\title{
In Response to: "Comparison of Prothrombin Time and Aspartate Aminotransferase in Predicting Hepatotoxicity After Acetaminophen Overdose."
}

\author{
Denise Fernandez $^{1}$ (i) $\cdot$ Lewis S. Nelson ${ }^{1}$ \\ Published online: 16 November 2015 \\ (C) American College of Medical Toxicology 2015
}

We read with great interest Levine et al.'s "Comparison of Prothrombin Time and Aspartate Aminotransferase in Predicting Hepatotoxicity After Acetaminophen Overdose." [1] With their patient population of confirmed acetaminophen exposures, the authors have the opportunity to shed light on this complicated toxin. However, their article raised several questions.

The author's research question is difficult to discern. Is their objective to determine the use of initial AST and PT as early predictors of hepatotoxicity or of fulminant hepatic failure? Today, many use the King's College criteria, among others, to predict who will develop fulminant hepatic failure from acetaminophen and require transfer to a liver transplant center [2]. However, the King's College criteria is limited in that none of the included clinical findings are early predictors. Therefore, if the authors' aim was to determine if AST and PT are better early predictors of disease, then obtaining information on the estimated timing of exposure was imperative. They define acetaminophen-induced hepatotoxicity as an AST> $1000 \mathrm{IU} / \mathrm{L}$, which is the definition used by convention, and they conclude that, "Initial AST performed better than the initial PT for predicting hepatic injury in this series of patients with APAP overdose." $[1,3]$ However, their results included 57 of 303 patients with an initial AST $>1000 \mathrm{IU} / \mathrm{L}$, biasing the prognostic ability of the test since they were already considered hepatotoxic. Relatedly, Table 1 shows a mean initial AST of $1208 \mathrm{IU} / \mathrm{L}$ with a median of $37 \mathrm{IU} / \mathrm{L}$, suggesting that their sample included a few outliers with significant hepatotoxicity at time of enrollment. To clarify any confusion, can the authors provide additional data to allow readers to independently understand their results. Perhaps an analysis excluding patients with elevated markers on initial assessment would be helpful.

\section{Conflict of Interest}

Dr. Nelson, an editorial board member of this journal, had no role in the peer review or publication decision of this letter or the article discussed in the letter.

\section{References}

1. Levine M, O'Connor AD, Padilla-Jones A, Gerkin RD. Comparison of prothrombin time and aspartate aminotransferase in predicting hepatotoxicity after acetaminophen overdose. J Med Toxicol 2015; published online 04 September:1-7.

2. O'Grady JG, Alexander GJM, Hayllar KM, Williams R. Early indicators of prognosis in fulminant hepatic failure. Gastroenterology. 1989;97(2):439-45.

3. Rumack BH, Peterson RG, Koch GG, Amara IA. Acetaminophen overdose. 662 cases with evaluation of oral acetylcysteine treatment. Arch Intern Med. 1981;141:380-5.
Denise Fernandez

denfernandez@gmail.com

1 Ronald O. Perelman Department of Emergency Medicine, New York School of Medicine, New York, NY, USA 